\title{
Manejo de la oferta forrajera en el Parque Nacional Campos del Tuyú y en campos ganaderos vecinos para la conservación del venado de las pampas
}

\author{
Adriana Rodríguez ${ }^{1, \bigotimes}$; Elizabeth Jacobo'; Germán Roitman'; Fernando Miñarro; \\ Pablo Preliasco ${ }^{2} \&$ Mario Beade ${ }^{3}$
}

${ }^{1}$ Facultad deAgronomía, Universidad deBuenos Aires, Argentina. ${ }^{2}$ Fundación VidaSilvestreArgentina. ${ }^{3}$ Administración de Parques Nacionales, Argentina.

\begin{abstract}
Resumen. El Parque Nacional Campos del Tuyú, ubicado en el extremo sur de la Bahía de Samborombón, es un área protegida creada para conservar el venado de las pampas (Ozotoceros bezoarticus celer). Los antecedentes sugieren que la protección del venado de las pampas dependería, entre otros factores, de la disponibilidad y calidad de la oferta forrajera provista por las dos comunidades preferidas como hábitat de alimentación: el monte de tala y el espartillar. Con el fin de mejorar la oferta de forraje se diseñaron y evaluaron prácticas de manejo específicas para las dos comunidades: a) exclusión del ganado en los montes de tala del establecimiento ganadero aledaño, luego de una defoliación intensa a fin de verano, y b) defoliaciones mecánicas periódicas en los espartillares. La defoliación intensa a fin del verano seguida por la exclusión al pastoreo del ganado entre marzo y agosto en los montes de tala del establecimiento vecino promovió la aparición o aumentó la contribución de los pastos nativos invernales Bromus catharticus, Bromus auleticus y Elymus scabrifolius, de los estivales Bothriochloa laguroides y Paspalum dilatatum y de la leguminosa Melilotus officinalis. La defoliación periódica del espartillar durante el período de crecimiento de la especie dominante, Spartina densiflora, incrementó la contribución de los pastos invernales nativos Elymus scabrifolius, Chaetotropis elongata y Phalaris angusta, y de la leguminosa Melilotus officinalis, y disminuyó la cobertura de la paja Cortaderia selloana. En ambas comunidades se registró un aumento del valor zootécnico ya que las especies que elevaron su contribución fueron pastos y leguminosas de alto valor nutritivo, mientras que las especies que disminuyeron su cobertura fueron herbáceas de escaso valor nutritivo.
\end{abstract}

[Palabras clave: disturbios, humedales, composición florística, calidad forrajera]

Aвstract. Management of forage offer in the National Park Campos del Tuyú and neighbouring cattle farms for Pampas's deer conservation. Campos del Tuyú National Park, located at the southern part of the Samborombón Bay, is a protected area created to promote the recovery of an endangered species, the pampas deer (Ozotoceros bezoarticus celer). Previous studies suggest that deer protection is related to the availability and nutritive value of forage provided by Celtis tala and Spartina densiflora communities, both preferred as feeding habitat. With the aim of enhace forage offer for pampa deer, specific management practices were designed: a) high intensity biomass defoliation in late summer followed by cattle exclusion from March to August in the Celtis community of the neighbor cattle farm, and b) periodic biomass defoliation of the Spartina densiflora Brongn community in the protected area. Defoliation in late summer followed by cattle exclusion from March to August in the Celtis community promoted the establishment or increased the contribution of the native cool-season grasses Bromus catharticus, Bromus auleticus and Elymus scabrifolius and warm-season grasses Bothriochloa laguroides and Paspalum dilatatum, and the legume Melilotus officinalis. Periodic defoliation of the Spartina densiflora community during its growing period increased the contribution of the native cool-season grasses Elymus scabrifolius, Chaetotropis elongata and Phalaris angusta and of the legume Melilotus officinalis, while decrease basal cover of the cordgrass Cortaderia selloana. Forage value increased in both communties because grasses and legumes species of high nutritive value increased their contribution, while herbaceous species of low nutritive value decrease their proportion.

[Keywords:disturbance, wetlands, floristic composition, forage quality]

Editora asociada: Ruggiero Adriana

arodrigu@agro.uba.ar
Recibido: 4 de septiembre de 2015

Aceptado: 26 de mayo de 2016 


\section{INTRODUCCIÓN}

Diversos modelos ecológicos han relacionado los disturbios con la conservación de la biodiversidad en distintos ecosistemas (Connell 1978; Pickett and White 1985; Milchunas et al. 1988; Westoby et al. 1989; Cingolani et al. 2005, 2008). En el contexto de estos modelos, se reconoce que los disturbios naturales y las prácticas tradicionales de manejo (e.g., cortes de biomasa de herbáceas o leñosas, pastoreo, quemas, entre otras) han sido factores relevantes para mantener la riqueza y la heterogeneidad de pastizales y humedales en todo el mundo (Middleton 1999; Rey Benayas et al. 2007). Sin embargo, es común que en las reservas naturales se eliminen los disturbios, lo que conduce a la disminución de la diversidad y la heterogeneidad del paisaje (Middleton 2013). Por otro lado, en áreas de uso agropecuario, no protegidas, la alta intensidad y/o frecuencia de disturbios antrópicos (e.g., cargas animales excesivas, pastoreo continuo, quemas o cortes frecuentes) también afectan de forma negativa los atributos de pastizales y humedales, lo que provoca cambios irreversibles en la vegetación y en los suelos (Cingolani et al. 2008).

El efecto de los disturbios sobre la dinámica de los ecosistemas depende del régimen de los disturbios, cuyos componentes son la frecuencia, la intensidad y la extensión espacial (Pickett and White 1985). Numerosos trabajos en ecosistemas pastoriles silvestres y manejados por el hombre han demostrado que el régimen de disturbio (combinaciones específicas de intensidad, frecuencia y escala espacial del pastoreo) modifica la composición florística, la productividad primaria y su estacionalidad, la composición química de los tejidos, los procesos biogeoquímicos, la diversidad de la fauna y flora nativa y la heterogeneidad del paisaje (McNaughton 1979, 1983; McNaughton et al. 1988; Biondini et al. 1988; Volesky et al. 2004; Patton et al. 2007; Augustine et al. 2012; Bylo et al. 2014). Sobre la base de estos conocimientos ecológicos se diseñan estrategias de manejo que, mediante el control de la frecuencia, intensidad y duración del período de pastoreo, procuran mejorar la condición de la vegetación y la receptividad del recurso forrajero, y optimizar la producción primaria y secundaria (Savory and Parsons 1980; Savory 1991; Heitschmidt and Taylor 1991; Deregibus et al. 1995).

Los pastizales pampeanos son comunidades florísticamente diversas (León et al. 1979;
León and Bertiller 1982; Burkart et al. 1989) que proveen importantes bienes y servicios ecosistémicos (Sala and Paruelo 1997; Bilenca and Miñarro 2004). Dado que esta región ha sufrido un importante proceso de transformación antrópica que comenzó hace más de 200 años y se intensificó en la última década, actualmente son escasos los sitios en los que persiste la vegetación original (León et al. 1984). Una de las subregiones donde aún predominan los pastizales naturales o semi-naturales es en la Pampa Deprimida (Vázquez and Rojas 2006). En estos pastizales, un disturbio de alta frecuencia y selectividad, como el pastoreo continuo, es considerado una de las principales causas del deterioro de la vegetación (Deregibus and Soriano 1981; Deregibus et al. 1995). Este tipo de pastoreo provocó el reemplazo de especies nativas perennes por dicotiledóneas y gramíneas anuales, en su mayoría exóticas y por lo general de escaso o nulo valor forrajero (Deregibus and Cahuepé 1983; Sala et al. 1986) y la drástica disminución de gramíneas invernales debido al sobrepastoreo inverno-primaveral de las áreas más altas del paisaje, donde predomina este grupo funcional (Jacobo et al. 2006). Por el contrario, el pastoreo controlado, que involucra la exclusión del pastoreo en momentos estratégicos y eventos de defoliación de intensidad y duración variables según la tasa de crecimiento de las especies clave, permite recuperar las especies de mayor valor forrajero en distintas comunidades de la región pampeana, en campos que habían estado sometidos a pastoreo continuo (Jacobo et al. 2006; Vecchio 2014). Así, un evento de pastoreo de alta intensidad y corta duración a fin de verano seguido de la exclusión al pastoreo durante el otoño promueve la germinación y establecimiento de los pastos anuales invernales y el macollaje de los pastos invernales perennes, lo cual incrementa la oferta forrajera invernal (Jacobo et al. 2000). Esta metodología de manejo sustentable ha sido probada a escala de establecimiento en diversas localidades de la Pampa Deprimida, $\mathrm{y}$ ha permitido conservar la biodiversidad florística e incrementar la producción de carne (Jacobo et al. 2006; Rodríguez and Jacobo 2012).

El objetivo general del presente trabajo fue validar la aplicación de distintas prácticas de manejo de pastizales pampeanos como herramienta para la conservación de una especie de mamíferos amenazada, el venado de las Pampas (Ozotoceros bezoarticus celer 
Cabrera 1943, categorizada como especie en peligro de extinción por Ojeda et al. 2012) en el Parque Nacional Campos del Tuyú. Esta área natural protegida está ubicada en el extremo sur de la Bahía de Samborombón, estuario externo del Río de La Plata (Partido de General Lavalle, Provincia de Buenos Aires, Argentina). Comprende 3000 ha, de las cuales $\sim 1 \%$ corresponde a un bosque xeromórfico subclimático (Cabrera and Zardini 1978) denominado "monte de tala", cuyo estrato herbáceo está dominado por pastos $\mathrm{C}_{3} \mathrm{y}_{4}$ de valor forrajero elevado (Fernández et al. 2004), mientras que un $50 \%$ del área está representado por la comunidad denominada "espartillar", dominada por especies del género Spartina en stands casi puros o asociadas a juncáceas, poáceas y otras herbáceas de porte más bajo (Bortolus 2006).

El venado de las pampas se alimenta preferentemente de los montes de tala y los espartillares recién quemados o pastoreados, y rechaza el resto de las comunidades y los espartillares no disturbados (Vila et al. 2008; Mentesana 2013). Como área de descanso prefiere los montes de tala, mientras que utiliza los espartillares altos, no disturbados, como refugio contra depredadores (en particular, las hembras y sus crías) (Vila et al. 1998). El venado selecciona los pastos y algunas dicotiledóneas que conforman el estrato herbáceo de los montes de tala (Merino 2003), especies también preferidas por el ganado vacuno (Deregibus and Cahuepé 1983). Si bien existe superposición en la dieta de ambas especies, la diferencia en el tamaño corporal y la estrategia de alimentación sugieren más bien facilitación que competencia por el forraje (Vila et al. 2008).

El pastoreo y el fuego fueron eliminados del Parque Nacional entrelos años 1979 y 1989 . Esto homogeneizó los espartillares, que quedaron constituidos por un estrato único dominado por matas altas de Spartina densiflora con más de $80 \%$ de material muerto. Si bien el número de venados de las pampas se incrementó en los primeros años, luego se redujo por migración hacia campos vecinos, lo que se atribuyó a la disminución de la oferta forrajera de mayor palatabilidad (Parera 2003; Fernández et al. 2004). En la actualidad, una solución habitual para este problema quemar los espartillares al final del verano, lo cual, sumado a la presencia de fuegos naturales, puede promover brotes nuevos que mejoren la calidad del forraje para el ganado (Vervoost 1967). Si bien las quemas generan una reducción de la cobertura y altura en lo inmediato, la comunidad se regenera rápidamente (Cagnoni and Faggi 1993; Cagnoni 1999; Nasca 2001). Asimismo, se ha demostrado que el fuego mejora la calidad de la oferta forrajera de los espartillares al eliminar el tejido senescente y promover el rebrote de tejido joven de $S$. densiflora, además de permitir el establecimiento de Melilotus sp., una leguminosa de gran valor nutritivo para el venado (Nasca 2001).

La remoción mecánica de la biomasa aérea del espartillar mediante cortes periódicos durante el período de crecimiento de $S$. densiflora, de octubre a mayo, tendría un efecto similar al fuego (Rodríguez et al. 2012). A su vez, la presencia de ganado afecta la selección del hábitat ya que los venados prefieren utilizar áreas sin ganado vacuno (Vila et al. 2008). Cuando el ganado es retirado de los establecimientos vecinos al parque, desde noviembre-diciembre hasta abril, los venados se desplazan hacia esos lotes (Vila et al. 2008). Esto refuerza el supuesto de facilitación en el consumo del forraje.

Los resultados obtenidos por Nasca (2001), Merino (2003), Vila et al. (2008) y Mentesana (2013) sugieren que la protección del venado de las pampas dependería, entre otros factores, de la disponibilidad y la calidad de la oferta forrajera provista por las dos comunidades preferidas: el monte de tala y el espartillar. Además, debido a la movilidad del venado hacia los campos vecinos una vez que se retira el ganado y al posible efecto de facilitación, una propuesta de manejo para mejorar la calidad y disponibilidad de la oferta forrajera debería incluir a los establecimientos ganaderos aledaños (Vila et al. 2008).

Con el fin de mejorar la oferta de forraje como una de las acciones de protección del venado de las pampas en el Parque Nacional Campos del Tuyú, este trabajo evalúa la efectividad de la implementación de distintas prácticas de manejo sobre los bosques de tala y espartillares en el Parque Nacional y en un establecimiento ganadero lindante (Las Tijeras), postulando las siguientes hipótesis: 1) una defoliación intensa a fin de verano seguida de la exclusión del ganado vacuno entre marzo y agosto en el establecimiento ganadero aledaño al parque incrementa la contribución de pastos nativos invernales en las lomas de tala. Se predice la aparición y el aumento de la cobertura relativadeBromuscatharticus,Bromusauleticus 
Trin. ex Nees y Elymus scabrifolius (Döll) J.H. Hunz. respecto de los stands mantenidos bajo pastoreo continuo, y 2) la defoliación periódica de la biomasa aérea del espartillar durante el período primavera-estivo-otoñal incrementa la contribución de los pastos nativos y de leguminosas invernales. Se predice la aparición y aumento de la cobertura relativa de Elymus scabrifolius y Melilotus officinalis en los stands cortados respecto de los stands no cortados. En virtud de los cambios florísticos esperados en ambas comunidades como resultado de las prácticas de manejo implementadas, se predice que aumentará el valor zootécnico del monte de tala y del espartillar.

\section{MATeRIALES y MÉTODOS}

\section{Área de estudio}

La Bahía de Samborombón es una planicie aluvial de 244000 ha. Conforma la franja oriental de la Pampa Deprimida. El clima es templado y húmedo, con precipitaciones de $1000 \mathrm{~mm} /$ año y una temperatura media anual de $15^{\circ} \mathrm{C}$, con máximas de $40^{\circ} \mathrm{C}$ y mínimas de $4^{\circ} \mathrm{C}$. Fue declarada "Humedal de Importancia Internacional" (Convención Ramsar 1997), "Refugio de Vida Silvestre" (Decreto de la Prov. de Buenos Aires N ${ }^{\circ}$ 12016/97), "Área Valiosa de Pastizal" y "Área de Importancia para la Conservación de las Aves" (Bilenca and Miñarro 2004) debido a la alta diversidad biológica que alberga.

El Parque Nacional Campos del Tuyú, de 3040 ha, y el establecimiento ganadero Las Tijeras (1650 ha) adyacente al Parque, están ubicados en el extremo sur de la Bahía de Samborombón (Partido de General Lavalle, Pcia. de Buenos Aires, $36^{\circ} 19^{\prime}-36^{\circ} 24^{\prime} \mathrm{S}$ y $56^{\circ} 49^{\prime}-57^{\circ} 55^{\prime} \mathrm{O}$ ). La Administración de Parques Nacionales estableció como objetivo específico del Parque Nacional Campos del Tuyú "la protección del venado de las Pampas y de los pastizales marginales costeros"(APN 2016).

Las comunidades vegetales de la Bahía de Samborombón responden a las características edáficas y a los regímenes de anegamientos, que a su vez dependen del relieve (Vervoorst 1967). Tanto en el Parque Nacional Campos del Tuyú como en el establecimiento Las Tijeras se presenta el mismo mosaico de comunidades. En las posiciones más altas del gradiente topográfico, sobre lomas convexas, altas, bien definidas y suelos muy bien drenados, se desarrolla el "monte de tala". Esta comunidad posee un estrato leñoso constituido por Celtis tala Gillies ex Planch y Scutia buxifolia Reissek, un estrato arbustivo formado por Jodina rhombifolia (Hook. \& Arn.) Reissek y Cestrum parquii L'Hér., y algunas enredaderas como Passiflora caerulea L. El estrato herbáceo está dominado por Bromus cathrarticus Vahl., Lolium multiflorumLam., Paspalum dilatatum Poir., Stenotaphrum secundatum (Walter) Kuntze, Melilotus officinalis (L.) Lam., entre otras herbáceas (Fernández et al. 2004). Esta comunidad ocupa sólo $1 \%$ de la superficie total del Parque y menos de 5\% de la superficie del establecimiento aledaño, y su estrato herbáceo representa la oferta de mayor valor forrajero para la alimentación del venado, en especial en invierno, cuando las otras comunidades no producen forraje. La comunidad más extendida es el "espartillar", que se desarrolla sobre suelos arcillosos y húmedos, ocupa las posiciones medias (0.8-1 m s.n.m.) y bajas (<0.4 m s.n.m.) del gradiente topográfico y recibe la influencia de los desbordes de las rías y canales de mareas. Está dominado por Spartina densiflora Brongn asociada a Juncus sp., Cortaderia selloana y a otras herbáceas de portemásbajocomoSarcocorniaperennis(Mill.) A.J.Scott, Distichlisspicata(L.)Greene, Malvella leprosa (Ortega) Krapov., Apium sellowianum H.Wolff, Limonium brasiliense(Boiss.) Kuntze, entre otras, en las posiciones medias y medias altas, y en stands casi puros en las posiciones más bajas (Di Bella et al. 2014a).

Mientras que el el pastoreo vacuno fue excluido del parque en 1979, en el establecimiento ganadero "Las Tijeras", así como en otros de la región, el ganado pastorea de manera continua los lotes donde coexisten los espartillares y los montes de tala durante otoño e invierno, cuando el rodeo de vacas de cría tiene requerimientos menores y hay agua de bebida disponible en las aguadas naturales. Dado que en estos lotes la superficie de montes de tala es muy inferior a la de los espartillares, el ganado ejerce una presión muy fuerte sobre el estrato herbáceo de los primeros.

\section{Aplicación de prácticas de manejo}

Se diseñaron prácticas de manejo específicas para las dos comunidades preferidas como hábitat de alimentación: a) defoliación intensa a fin de verano seguida de exclusión del ganado en los montes de tala del establecimiento ganadero aledaño, y b) defoliaciones mecánicas periódicas en los espartillares. 
ManejodelosMontes de Tala. Para determinar si la defoliación intensa de la biomasa a fin de verano seguido por la exclusión al pastoreo vacuno entre marzo y agosto incrementa la contribución de pastos nativos invernales en los montes de tala pastoreadas de forma continua, en el establecimiento ganadero "Las Tijeras", adyacente al Parque Nacional Campos del Tuyú, en otoño de 2010 se delimitaron con alambrado eléctrico áreas de 1 a 2 ha que incluían stands de montes de tala, mientras otras permanecieron con libre acceso del ganado (Figura 1). En las áreas cercadas se cortó mecánicamente la biomasa a fin de febrero o principios de marzo, se impidió el acceso del ganado hasta agosto, y luego se permitió el pastoreo vacuno hasta octubre. Este manejo se repitió durante cuatro años consecutivos en las áreas cercadas, mientras que las áreas no cercadas se mantuvieron bajo pastoreo continuo.

Se seleccionaron tres stands de monte de tala dentro de las áreas cercadas, bajo el tratamiento de defoliación intensa a fin de verano y exclusión del pastoreo vacuno entre marzo y agosto, y otros tres stands en las áreas con libre acceso del ganado, bajo el tratamiento de pastoreo continuo. En estos stands se relevó la composición florística en abril y octubre de los años 2010, 2012 y 2013. La cobertura basal de cada especie se estimó mediante el método del punto al paso (Mueller-Dombois and Ellemberg 1974) a lo largo de tres transectas no permanentes de $5 \mathrm{~m}$ de longitud, relevando 100 puntos por transecta. Se agruparon las especies según grupos funcionales (Lavorel et al. 1997) y se estimó la cobertura basal de cada grupo.

En cada stand se estimó el Valor Zootécnico utilizando la ecuación propuesta por Daget and Poissonet (1971):

$\mathrm{FQI}=\sum \mathrm{p}_{\mathrm{i}}^{*} \mathrm{q}_{\mathrm{i}}$

donde $\mathrm{p}_{\mathrm{i}}$ es la proporción de cada especie en la muestra y $\mathrm{q}_{\mathrm{i}}$ es el valor de calidad específico para cada especie. Cahuepé et al. (1985) propusieron valores de $\mathrm{q}_{\mathrm{i}}$ para los pastizales de la Pampa Deprimida; los mismos oscilan entre 0 (sin valor forrajero) y 5 (excelente calidad forrajera) para el ganado vacuno. Se utilizaron los mismos valores para el venado debido a que coinciden en gran medida las especies seleccionadas (cfr. Deregibus and Cahuepé 1983 con Merino 2003).
Para caracterizar las principales propiedades químicas de los suelos, de cada stand se extrajeron 5 submuestras de suelo $(4 \mathrm{~cm}$ de diámetro y $12 \mathrm{~cm}$ de profundidad) al inicio (otoño 2010) y al final del período experimental (primavera 2013). Las submuestras fueron secadas al aire, tamizadas por un tamiz de $2 \mathrm{~mm}$ y se compusieron en una muestra compuesta para determinar el contenido de carbono orgánico (CO) por el método de Walkley and Black y el $\mathrm{N}$ total por el método Kjeldahl. Se analizo además el pH y conductividad eléctrica. Estos análisis mostraron que las características de los suelos en ambos tratamientos y fechas de muestreo resultaron similares. Los valores medios fueron: materia orgánica (\%) 3.42 \pm 0.19 ; nitrógeno (\%) 0.23 \pm 0.01 , fósforo (ppm) $16.02 \pm 8.07, \mathrm{pH} 7.01 \pm 0.52$ y conductividad eléctrica $(\mathrm{dS} / \mathrm{m}) \quad 0.47 \pm 0.12$.

Manejo del espartillar. Para determinar si la defoliación periódica del espartillar en el período primavero-estivo-otoñal incrementa la contribución de pastos y leguminosas invernales, se seleccionaron en el Parque Nacional Campos del Tuyú tres stands en la posición topográfica alta de la comunidad dado que los cambios en la vegetación por disturbios como el pastoreo ocurren de manera más intensa en las posiciones relativamente altas del espartillar (Di Bella et al. 2014a). Dentro de cada stand se delimitaron dos parcelas de $5 \times 5 \mathrm{~m}$. A una de las parcelas de cada stand se le aplicaron cortes mecánicos con motoguadaña manual a $25 \mathrm{~cm}$ de altura cada 60 días entre octubre y mayo, durante tres ciclos de crecimiento (2010-2011, 2011-2012 y 2012-2013). La otra parcela de cada stand no fue cortada (Figura 1). La altura de corte se determinó mediante mediciones previas de la altura remanente de $S$. densiflora en establecimientos vecinos bajo pastoreo. En cada parcela se relevó la composición florística en los meses de diciembre y mayo de cada año. Luego se agruparon las especies por grupos funcionales y se calculó el valor zootécnico siguiendo los mismos procedimientos descriptos para las montes de tala.

\section{Precipitaciones}

La escala temporal de los experimentos permitió considerar la variación interanual de las precipitaciones. Las precipitaciones en los años 2010 y 2012 superaron la media histórica (967 mm/año): 1015 y 1242 mm/ año, respectivamente. Por su parte, las 


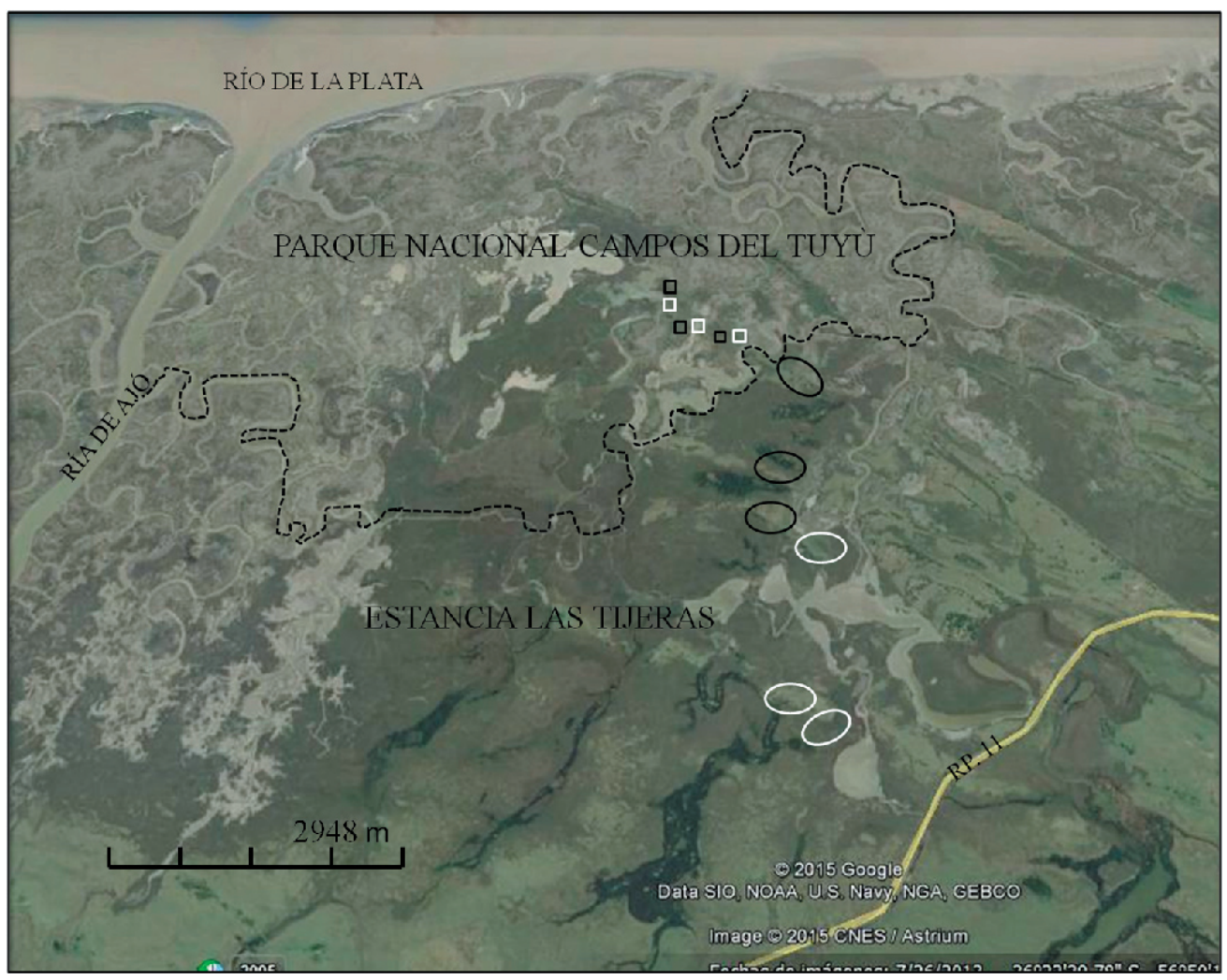

Figura 1. Imagen satelital del Parque Nacional Campos del Tuyú y de la Estancia Las Tijeras. Los óvalos indican los stands de Montes de Tala (blancos=stands bajo pastoreo continuo; negros=stands bajo el tratamiento de defoliación intensa a fin de verano y exclusión del pastoreo entre marzo y agosto). Los cuadrados indican las parcelas en stands de la posición topográfica alta del espartillar (negros=parcelas control no defoliadas; blancos=parcelas defoliadas cada 60 días entre octubre y mayo). Imagen obtenida de Google Earth, Data SIO NOAA US Navy NGA GEBCO. Fecha de la imagen: 7 de junio de 2013.

Figure 1. Satelital image of Campos del Tuyú National Park and Las Tijeras farm. Ovals indicate Celtis ehrenbergiana stands (white=stands under continuous cattle grazing; black=stands under late summer defoliation followed by cattle exclusion from March to August). Squares indicate plots in the Spartina densiflora upper position stands (black=notdefoliated control treatment; white=defoliation each 60 days from October to March treatment). Image taken from Google Earth, Data SIO NOAA US Navy NGA GEBCO. Image date: June 7, 2013.

precipitaciones registradas en los años 2011 y 2013 resultaron inferiores a la media: 673 y $626 \mathrm{~mm} /$ año, respectivamente.

\section{Análisis estadísticos}

Para determinar diferencias en la composición florística de la vegetación de la monte de tala y del espartillar por efecto de los tratamientos aplicados en cada período de medición se utilizó la prueba MRPP (Multi-Response Permutation Procedure) (Biondini et al. 1988b), y para identificar las especies responsables de las diferencias entre tratamientos se aplicó el Análisis de Especies Indicadoras y la prueba Monte Carlo (Dufrene and Legendre 1997). Se usaron los datos de frecuencia de especies (medidas relativas, ponderadas por sus totales) en cada unidad experimental (stand o parcela). Para evitar darle demasiada relevancia a las especies con baja abundancia en las matrices de datos, se excluyeron aquellas cuya constancia fue menor a $5 \%$.

La variable cobertura relativa de cada grupo funcional fue transformadas angularmente (arco seno de la raíz cuadrada) para cumplir con el supuesto de homogeneidad del análisis de varianza. El efecto de los tratamientos sobre esta variable, sobre el índice de calidad forrajera y sobre las propiedades químicas del suelo se analizó mediante un análisis de varianza con un arreglo de medidas repetidas, tomando como efecto intra-sujeto (medidas repetidas) los períodos de medición. Cuando el efecto intra-sujeto y su interacción no resultaron significativos, se promediaron los datos de los períodos de medición; cuando fueron significativos, se compararon las medias mediante la prueba de Tukey $(P<0.05)$ o se calcularon los contrastes de interés. 


\section{Resultados}

\section{Manejo de los Montes de Tala}

Cobertura relativa de los grupos funcionales. La defoliación intensa a fin de verano seguida de exclusión al pastoreo del ganado entre marzo y agosto modificó la cobertura relativa de todos los grupos funcionales (Figura 2).
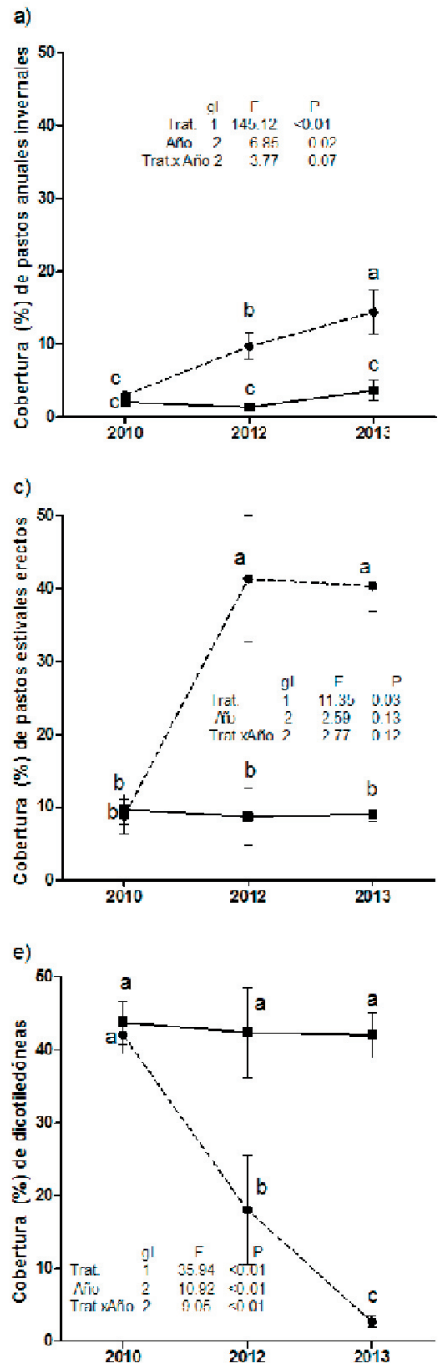

La cobertura basal de los pastos anuales invernales se incrementó de manera gradual con la defoliación intensa a fin de verano seguida de la exclusión del pastoreo vacuno (Figura 2a), en tanto los pastos perennes invernales y las leguminosas incrementaron su contribución pero más tardíamente, a partir del tercer ciclo de exclusión del pastoreo entre marzo y agosto (Figura $2 b$ y f). La cobertura
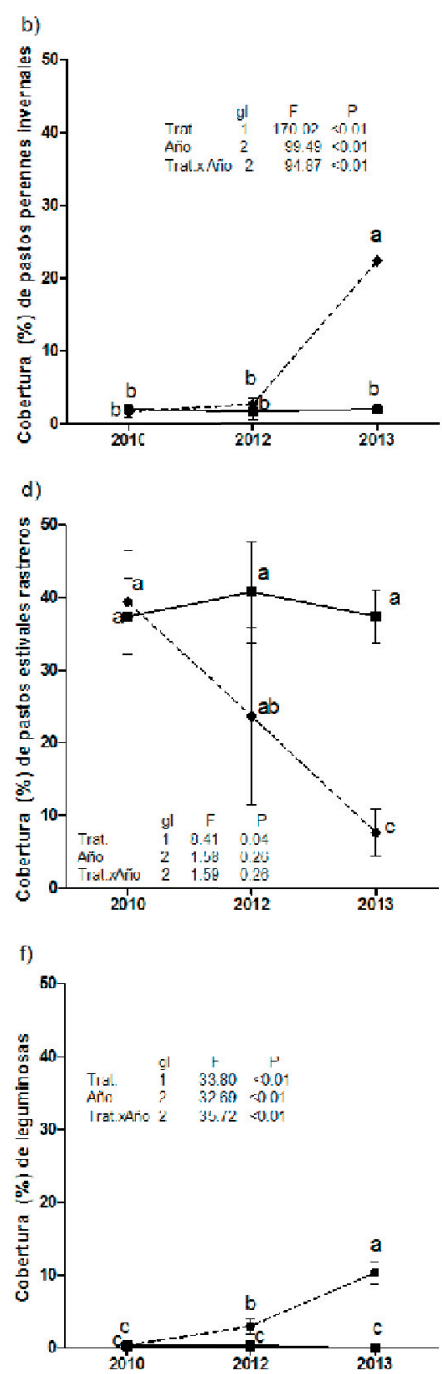

Figura 2. Evolución de la cobertura basal relativa (\%) de los grupos funcionales en los stands de monte de tala bajo el tratamiento de pastoreo continuo (línea llena) y el tratamiento de defoliación intensa a fin de verano seguida de exclusión del pastoreo entre marzo y agosto (línea punteada) durante cuatro años consecutivos desde el inicio del tratamiento. Las barras verticales indican un error estándar $(1 \mathrm{EE})$ de la media. Se insertaron los resultados del análisis de varianza con un arreglo de medidas repetidas: $F$, grados de libertad $(\mathrm{gl})$ y $P$ para el factor principal tratamiento (Trat.), efecto intra-sujeto (medida repetida Año) e interacción entre el factor principal y el efecto intra-sujeto (Trat. $\mathrm{x}$ Año). Letras distintas indican diferencias significativas surgidas de la prueba de Tukey.

Figure 2. Relative basal cover (\%) of functional groups in Celtis tala stands under continuous cattle grazing treatment (full line) and in the late summer defoliation followed by cattle exclusion from March to August treatment (dotted line). Vertical lines indicate one standard error (1 SE) of the mean. Insert: values of the repeated-measures analysis of variance: $F$, degree of freedom ( $\mathrm{gl})$ and $P$ for the main factor (Trat.), within-subject factor (repeated measure Año) and interaction between the main and the within-subject factors (Trat. $x$ Año). Different letters mean significant differences obtained by Tukey test 
Tabla 1. Cobertura basal (\%) de las especies en los stands de monte de tala bajo el tratamiento de pastoreo continuo (PC) y el tratamiento de defoliación intensa a fin de verano seguida de exclusión del pastoreo entre marzo y agosto (D+EP). Características de las especies: $\mathrm{CV}=$ ciclo de vida de cada especie (A: anuales, $\mathrm{B}$ : bianuales, $\mathrm{P}$ : perennes), $\mathrm{O}=$ origen ( $\mathrm{N}$ : nativas, E: exóticas) y $\mathrm{Q}=$ valor zootécnico. Los valores son las medias de los stands y los errores estándar de las medias se muestran entre paréntesis. Los estadísticos VI (valores de indicación, como porcentaje de indicación perfecta) y P resultan del Análisis de Especies Indicadoras de la prueba Monte Carlo para el año 2013.

Table 1. Basal cover (\%) of species for the Celtis tala stands in the continuous cattle grazing treatment (PC) and in the late summer defoliation followed by cattle exclusion from March to August treatment (D+EP). Species traits: $C V=l i f e$ cycle (A: annual, B: biannual, $\mathrm{P}$ : perennial), $\mathrm{O}=$ origin (N: native, $\mathrm{E}$ : exotic) and $\mathrm{Q}$ : forage quality). Values are means of stands and standard errors of means are shown within parentheses. Statistics VI (indication values, as percentage of perfect indication) and P result of Monte Carlo test of significance of Indicator Species Analysis for year 2013.

\begin{tabular}{|c|c|c|c|c|c|c|c|c|c|}
\hline \multirow[t]{2}{*}{ Grupo funcional/Especies } & \multicolumn{3}{|c|}{ Características } & \multicolumn{4}{|c|}{ Tratamientos - Años } & \multirow[b]{2}{*}{ VI } & \multirow[b]{2}{*}{$P$} \\
\hline & $\mathrm{CV}$ & $\mathrm{O}$ & Q & PC 2010 & D+EP 2010 & PC 2013 & D+EP 2013 & & \\
\hline \multicolumn{10}{|l|}{ Pastos anuales invernales } \\
\hline Bromus catharticus Vahl & $\mathrm{A} / \mathrm{B}$ & $\mathrm{N}$ & 5 & $1.08(0.72)$ & $1.25(0.43)$ & $1.33(1.53)$ & $6.33(3.21)$ & 83 & $<0.01^{*}$ \\
\hline $\begin{array}{l}\text { Lolium multiflorum Lam. } \\
\text { Pastos perennes invernales }\end{array}$ & A & $\mathrm{E}$ & 5 & $0.66(0.41)$ & $1.33(1.02)$ & $2.33(2.31)$ & $8.00(2.00)$ & 77 & $<0.01^{*}$ \\
\hline Bromus auleticus Trin. ex Nees & $\mathrm{P}$ & $\mathrm{N}$ & 5 & & & & $12.00(3.00)$ & 100 & $<0.01^{*}$ \\
\hline Elymusscabrifolius(Döll)J.H.Hunz & $\mathrm{P}$ & $\mathrm{N}$ & 3 & & & & $2.67(2.08)$ & 100 & $<0.01^{*}$ \\
\hline $\begin{array}{l}\text { Jarava plumosa (Spreng.) S.W.L. } \\
\text { Jacobs and J. Everett }\end{array}$ & $\mathrm{P}$ & $\mathrm{N}$ & 2 & $1.17(1.04)$ & $0.67(0.76)$ & $1.33(1.53)$ & $3.33(3.51)$ & 47 & 0.79 \\
\hline Nassella hyalina (Nees) Barkworth & $\mathrm{P}$ & $\mathrm{N}$ & 2 & $0.78(1.34)$ & $1.11(0.98)$ & $0.67(1.15)$ & $4.33(3.06)$ & 86 & 0.18 \\
\hline Pastos estivales erectos & & & & & & & & & \\
\hline Bothriochloalaguroides(DC.)Herter & $\mathrm{P}$ & $\mathrm{N}$ & 4 & $0.15(0.26)$ & $0.17(0.25)$ & & $14.67(3.51)$ & 100 & $<0.01^{*}$ \\
\hline Eleusine indica (L.) Gaertn. & A & $\mathrm{E}$ & 2 & $0.57(0.98)$ & $1.07(1.09)$ & $1.00(1.73)$ & $1.33(1.01)$ & 33 & 1.00 \\
\hline Eleusine tristachya (Lam.) Lam. & $P$ & $\mathrm{~N}$ & 2 & $4.96(1.86)$ & $3.46(3.69)$ & $4.67(4.51)$ & $2.67(2.08)$ & 42 & 1.00 \\
\hline Paspalum dilatatum Poir & $\mathrm{P}$ & $\mathrm{N}$ & 4 & $0.99(1.71)$ & $0.99(1.49)$ & & $17.33(5.03)$ & 100 & $<0.01^{*}$ \\
\hline Setaria parviflora (Poir.) Kerguélen & $\mathrm{P}$ & $\mathrm{N}$ & 2 & $1.62(0.77)$ & $2.12(0.93)$ & $3.33(1.53)$ & $2.33(2.08)$ & 58 & 0.70 \\
\hline Sporobulus indicus (L.) R. Br. & $\mathrm{P}$ & $\mathrm{N}$ & 3 & $0.98(1.70)$ & $1.09(0.85)$ & & $2.33(2.52)$ & 66 & 0.39 \\
\hline \multicolumn{10}{|l|}{ Pastos estivales rastreros } \\
\hline Cynodon dactylon (L.) Pers & $\mathrm{P}$ & $\mathrm{E}$ & 2 & $22.12(8.80)$ & $22.46(12.45)$ & $14.00(3.61)$ & $2.00(2.65)$ & 88 & $<0.01^{*}$ \\
\hline Distichlis spicata (L.) Greene & $\mathrm{P}$ & $\mathrm{N}$ & 1 & $3.17(3.55)$ & $4.33(1.76)$ & $8.67(3.51)$ & $1.00(1.66)$ & 90 & $<0.01^{*}$ \\
\hline Paspalum vagunatum Sw. & $\mathrm{P}$ & $\mathrm{N}$ & 2 & 4 & 3) & $06)$ & 2. & 76 & 0.18 \\
\hline Stenotephrumsecundatum(Walter) & $\mathrm{P}$ & $\mathrm{N}$ & 2 & $7.14(2.72)$ & $8.14(2.90)$ & $6.33(4.16)$ & $2.00(1.73)$ & 76 & 0.29 \\
\hline \multicolumn{10}{|l|}{ Kuntze $\quad$ Leguminosas } \\
\hline Medicago lupulina L. & $\mathrm{A} / \mathrm{B}$ & $\mathrm{E}$ & 4 & $0.17(0.29)$ & $0.15(0.25)$ & & & & \\
\hline Melilotus officinalis (L.) Lam. & $\mathrm{A} / \mathrm{B}$ & $\mathrm{E}$ & 4 & & & & $10.33(2.52)$ & 100 & $<0.01^{*}$ \\
\hline \multicolumn{10}{|l|}{ Dicotiledóneas } \\
\hline Ambrosia tenuifolia Spreng. & $\mathrm{P}$ & $\mathrm{N}$ & 0 & $10.71(3.33)$ & $9.55(6.32)$ & $9.67(4.73)$ & $0.33(0.58)$ & 97 & $<0.01^{*}$ \\
\hline Baccharis glutinosa Pers. & $\mathrm{P}$ & $\mathrm{N}$ & 0 & & & & $0.33(0.58)$ & 33 & 1.00 \\
\hline Bupleurum tenuissimum L. & A & $\mathrm{E}$ & 0 & & & & $0.33(0.58)$ & 33 & 1.00 \\
\hline Centaurea melitensis L. & $\mathrm{A} / \mathrm{B}$ & $\mathrm{E}$ & 0 & $13.24(1.65)$ & $13.91(3.00)$ & $15.00(4.00)$ & & 100 & $<0.01^{*}$ \\
\hline $\begin{array}{l}\text { Conyza bonariensis (L.) Cronquist } \\
\text { var. bonariensis }\end{array}$ & $\mathrm{A}$ & $\mathrm{N}$ & 0 & $2.64(4.58)$ & 2.94( & & & & \\
\hline Dichondra microcalyx (Hallier f.) & $\mathrm{P}$ & $\mathrm{N}$ & 0 & $1.79(1.55)$ & $1.99(1.78)$ & & $0.67(0.58)$ & 67 & 0.39 \\
\hline $\begin{array}{l}\text { Fabris } \\
\text { Malvella leprosa (Ortega) Krapov. }\end{array}$ & $\mathrm{P}$ & $\mathrm{N}$ & 0 & $5.56(3.81)$ & $5.06(0.86)$ & $8.33(2.08)$ & & 100 & $<0.01^{*}$ \\
\hline Phyla canescens (Kunth) Greene & $\mathrm{P}$ & $\mathrm{N}$ & 0 & $8.84(1.44)$ & $8.18(4.27)$ & $9.00(2.00)$ & $0.33(0.58)$ & 96 & $<0.01^{*}$ \\
\hline Physalis viscosa L. & $\mathrm{P}$ & $\mathrm{N}$ & 1 & $0.84(1.23)$ & $1.01(1.55)$ & & & & \\
\hline Plantago lanceolata L. & $\mathrm{P}$ & $\mathrm{E}$ & 2 & $0.16(1.14)$ & $0.17(0.29)$ & & $0.67(0.58)$ & 67 & 0.39 \\
\hline \multicolumn{10}{|l|}{ Ciperáceas } \\
\hline $\begin{array}{l}\text { Juncus balticus Willd. ssp. andicola } \\
\text { (Hook.) Snogerup }\end{array}$ & $\mathrm{P}$ & $\mathrm{N}$ & 1 & $0.08(0.15)$ & $0.11(0.33)$ & & $0.33(0.58)$ & 33 & 1.00 \\
\hline Care & $\mathrm{P}$ & $\mathrm{N}$ & 1 & $0.71(0.83)$ & $0.85(1.27)$ & & $1.00(1.00)$ & 67 & 0.39 \\
\hline
\end{tabular}


basal de los pastos estivales erectos se incrementó con la exclusión del pastoreo vacuno entre marzo y agosto (Figura 2c). Por el contrario, tanto los pastos estivales rastreros (Figura 2d) como las dicotiledóneas (Figura 2e) disminuyeron de forma sostenida su cobertura basal a medida que se sucedieron los ciclos de exclusión del pastoreo vacuno.

Composición florística específica. La composición florística específica antes de establecer los tratamientos (año 2010) no resultó diferente entre ellos ( $T=1.33, P=0.90)$. Las diferencias en la composición florística se expresaron en los relevamientos del año 2012 $(T=-2.07, P=0.03)$ y $2013(T=-2.70, P=0.02)$. Las especies responsables de las diferencias entre los stands mantenidos bajo pastoreo continuo y los que fueron defoliados intensamente a fin de verano, antes de la exclusión del pastoreo entre marzo y agosto (identificadas a través de análisis de especies indicadoras y la prueba de Monte Carlo) fueron los pastos invernales anualesBromuscatharticusy Loliummultiflorum, lospastosinvernalesperennes Bromusauleticus y Elymus scabrifolius, los pastos estivales erectos Bothriochloa laguroides y Paspalum dilatatum y la leguminosa Melilotus officinalis, que aumentaron su cobertura relativa con la exclusión del pastoreo entre marzo y agosto (Tabla 1). También contribuyeron a las diferencias en la composición florística los pastos estivales rastreros Cynodon dactylon y Distichlis spicatay las dicotiledóneas Ambrosia tenuifolia,Centaureamelitensis,Malvellaleprosa y Phyla canescens, que redujeron su cobertura relativa con la exclusión del pastoreo entre marzo y agosto (Tabla 1).

Valor forrajero. La exclusión del pastoreo entre marzo y agosto incrementó sostenidamente el valor forrajero de la comunidad, que se triplicó luego de cuatro períodos sucesivos de este manejo (Figura 3).

\section{Espartillares}

Cobertura relativa de las especies dominantes y grupos funcionales. El efecto de las defoliaciones durante el período de crecimiento en la cobertura basal de $S$. densiflora y de los otros grupos funcionales se muestra en la Figura 4. La especie dominante, S. densiflora, mantuvo constante su cobertura basal bajo defoliaciones periódicas, mientras que el testigo no defoliado aumentó la cobertura basal sólo en el año 2011 (Figura 4a). El grupo funcional de las pajas mantuvo una cobertura constante y menor a $10 \%$ bajo

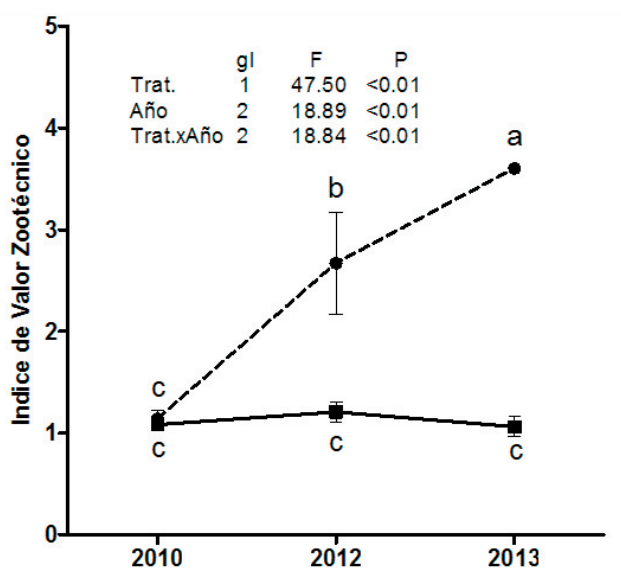

Figura 3. Valor forrajero de la vegetación de la comunidad de monte de tala en los stands bajo el tratamiento de pastoreo continuo (línea entera) y el tratamiento de defoliación intensa a fin de verano seguida de exclusión del pastoreo entre marzo y agosto (líneas punteadas). Las barras verticales indican un error estándar (1 EE) de la media. Se insertaron los resultados del análisis de varianza con un arreglo de medidas repetidas: F, grados de libertad (gl) y P para el factor principal tratamiento (Trat.), efecto intra-sujeto (medida repetida Año) e interacción entre el factor principal y el efecto intra-sujeto (Trat. x Año). Letras distintas indican diferencias significativas surgidas de la prueba de Tukey.

Figure 3. Forage quality index in the Celtis tala stands under continuous cattle grazing treatment (full line) and in the late summer defoliation followed by cattle exclusion from March to August treatment (dotted line). Vertical lines indicate one standard error (1 SE) of the mean. Insert: values of the repeated-measures analysis of variance: $\mathrm{F}$, degree of freedom $(\mathrm{gl})$ and $\mathrm{P}$ for the main factor (Trat.), within-subject factor (repeated measure Año) and interaction between the main and the within-subject factors (Trat. x Año). Different letters mean significant differences obtained by Tukey test.

defoliaciones, en tanto que se incrementó de manera lineal a partir del año 2011 en el testigo no defoliado (Figura $4 \mathrm{~b}$ ). Los pastos invernales perennes respondieron en forma opuesta a las pajas, ya que en ausencia de defoliaciones, la cobertura basal se mantuvo en valores inferiores a 3\%, mientras que bajo defoliaciones periódicas la cobertura basal se incrementó hasta 30\% (Figura 4c). La cobertura de los pastos estivales rastreros, que tuvo su máximo valor (promedio 28\%) al inicio del período experimental en ambos tratamientos, fue disminuyendo gradualmente hasta alcanzar valores mínimos (11\%) en el año 2013 tanto en ausencia de defoliaciones como bajo defoliaciones periódicas (Figura 4d). La cobertura basal de las dicotiledóneas mostró una tendencia a ser inferior bajo defoliaciones periódicas respecto del tratamiento testigo no defoliado a lo largo de todo el período 
Tabla 2. Cobertura basal (\%) de las especies en los stands de espartillares bajo el tratamiento de defoliaciones cada 60 días entre octubre y mayo (D O-M) y el testigo no defoliado (ND) al inicio (año 2010) y al final (año 2013) del período experimental. Características de las especies: $\mathrm{CV}=$ ciclo de vida de cada especie (A: anuales, B: bianuales, P: perennes), $\mathrm{O}=$ origen ( $\mathrm{N}$ : nativas, E: exóticas) y $\mathrm{Q}=$ valor zootécnico. Los valores son las medias de los stands y los errores estándar de las medias se muestran entre paréntesis. Los estadísticos VI (valores de indicación, como porcentaje de indicación perfecta) y P resultan del Análisis de Especies Indicadoras de la prueba Monte Carlo para el año 2013.

Table 2. Basal cover (\%) of species for the Spartina densiflora stands in the defoliation each 60-days from October to March treatment (D O-M) and in the not-defoliated control treatment (ND) at the beginning (2010) and at the end (2013) of the experimental period. Species traits: $C V=$ life cycle (A: annual, B: biannual, P: perennial), $\mathrm{O}=$ origin $(\mathrm{N}$ : native, E: exotic) and Q: forage quality). Values are means of stands and standard errors of means are shown within parentheses. Statistics VI (indication values, as percentage of perfect indication) and P result of Monte Carlo test of significance of Indicator Species Analysis for year 2013.

\begin{tabular}{|c|c|c|c|c|c|c|c|c|c|}
\hline \multirow[t]{2}{*}{ Grupo funcional/Especies } & \multicolumn{4}{|c|}{ Características } & \multicolumn{3}{|c|}{ Tratamientos - Años } & \multirow[b]{2}{*}{ VI } & \multirow[b]{2}{*}{$P$} \\
\hline & $\mathrm{CV}$ & $\mathrm{O}$ & $\mathrm{Q}$ & D O M 2010 & ND 2010 & D O M 2013 & ND 2013 & & \\
\hline Spartina densiflora Brongn & $\mathrm{P}$ & $\mathrm{N}$ & 2 & $28.8(2.8)$ & $32.5(4.8)$ & $30.4(6.7)$ & $30.0(4.6)$ & 50 & 1.00 \\
\hline \multicolumn{10}{|l|}{ Pastos estivales } \\
\hline Distichlis spicata (L.) Greene & $\mathrm{P}$ & $\mathrm{N}$ & 1 & $29.8(7.3)$ & $23.7(15.0)$ & $4.7(4.6)$ & $3.0(3.6)$ & 61 & 0.59 \\
\hline Cynodon plectostachyus (K. & $\mathrm{P}$ & $\mathrm{E}$ & 4 & $2.0(2.6)$ & $1.2(1.6)$ & $7.3(4.7)$ & $7.8(6.3)$ & 51 & 1.00 \\
\hline \multicolumn{10}{|l|}{$\begin{array}{l}\text { Schum.) Pilg. } \\
\text { Pastos invernales }\end{array}$} \\
\hline Poa trivialis L. & $\mathrm{P}$ & $\mathrm{E}$ & 4 & $0.7(1.2)$ & $0.5(0.9)$ & $2.0(1.0)$ & $0.3(0.5)$ & 86 & 0.20 \\
\hline Polypogon monspeliensis (L.) & A & $\mathrm{E}$ & 3 & $0.3(0.3)$ & $0.8(0.8)$ & $1.0(1.0)$ & & 67 & 0.40 \\
\hline $\begin{array}{l}\text { Desf. } \\
\text { Hordeum stenostachys Godr. }\end{array}$ & $\mathrm{P}$ & $\mathrm{N}$ & 2 & $0.5(0.9)$ & & & & & \\
\hline Elymus scabrifolius (Döll) J.H. & $\mathrm{P}$ & $\mathrm{N}$ & 3 & & $0.3(0.6)$ & $14.0(2.6)$ & & 100 & $<0.05^{*}$ \\
\hline $\begin{array}{l}\text { Hunz. } \\
\text { Chaetotropis elongata (Kunth) }\end{array}$ & A & $\mathrm{N}$ & 3 & & & $7.3(2.1)$ & $1.0(1.0)$ & 100 & $<0.05^{*}$ \\
\hline $\begin{array}{l}\text { Björkman } \\
\text { Phalaris angusta Nees ex Trin. }\end{array}$ & A & $\mathrm{N}$ & 4 & & & $8.7(1.5)$ & $1.0(1.0)$ & 90 & $<0.05^{*}$ \\
\hline \multicolumn{10}{|l|}{ Pajas } \\
\hline $\begin{array}{l}\text { Cortaderia selloana (Schult. and } \\
\text { Schult. f.) Asch. and Graebn. }\end{array}$ & $\mathrm{P}$ & $\mathrm{N}$ & 1 & & $1.5(1.3)$ & $5.1(4.4)$ & $26.7(20.2)$ & 84 & $<0.05^{*}$ \\
\hline $\begin{array}{l}\text { Phragmites australis (Cav.) Trin. } \\
\text { ex Steud. }\end{array}$ & P & $\mathrm{N}$ & 1 & & $0.5(0.9)$ & & & & \\
\hline \multicolumn{10}{|l|}{ Ciperáceas } \\
\hline Cyperus eragrostis Lam. & $\mathrm{P}$ & $\mathrm{N}$ & 0 & $0.7(1.2)$ & $1.5(1.8)$ & & & & \\
\hline Carex bonariensis Desf. ex Poir. & $\mathrm{P}$ & $\mathrm{N}$ & 2 & $3.7(6.4)$ & $4.3(6.7)$ & & $1.0(1.7)$ & 33 & 1.00 \\
\hline $\begin{array}{l}\text { Juncus balticus Willd. ssp. } \\
\text { andicola (Hook.) Snogerup }\end{array}$ & $\mathrm{P}$ & $\mathrm{N}$ & 2 & $0.5(0.9)$ & $1.2(1.6)$ & & $6.0(5.3)$ & 67 & 0.40 \\
\hline Leguminosas & & & & & & & & & \\
\hline $\begin{array}{l}\text { Melilotus officinalis (L.) Lam. } \\
\text { Dicotiledóneas }\end{array}$ & $\mathrm{A} / \mathrm{B}$ & $\mathrm{E}$ & 4 & $4.2(5.1)$ & $2.3(2.5)$ & $9.7(2.1)$ & $0.7(1.2)$ & 94 & $<0.05^{*}$ \\
\hline $\begin{array}{l}\text { Sarcocornia perennis (Mill.) A. } \\
\text { J.Scott }\end{array}$ & $\mathrm{P}$ & $\mathrm{E}$ & 1 & $11.2(10.5)$ & $5.5(5.9)$ & $2.7(2.1)$ & $5.0(2.6)$ & 65 & 0.39 \\
\hline Baccharis pingraea DC. & $\mathrm{P}$ & $\mathrm{N}$ & 0 & $6.0(2.3)$ & $12.0(10.8)$ & $3.3(1.5)$ & $3.7(4.0)$ & 48 & 0.90 \\
\hline $\begin{array}{l}\text { Aster squamatus (Spreng.) } \\
\text { Hieron. }\end{array}$ & $\mathrm{P}$ & $\mathrm{N}$ & 0 & $0.5(0.9)$ & $1.3(2.3)$ & & & & \\
\hline Senecio selloi (Spreng.) DC. & $\mathrm{P}$ & $\mathrm{E}$ & 0 & $0.5(0.9)$ & $1.3(1.5)$ & & & & \\
\hline Centaurea calcitrapa L. & $\mathrm{A} / \mathrm{B}$ & $\mathrm{E}$ & 0 & $1.5(1.3)$ & $0.8(0.6)$ & & & & \\
\hline Ambrosia tenuifolia Spreng. & $\mathrm{P}$ & $\mathrm{N}$ & 0 & $1.8(3.2)$ & $1.3(2.3)$ & $3.0(1.7)$ & $6.3(5.5)$ & 45 & 1.00 \\
\hline Solanum bonaeriense L. & $\mathrm{P}$ & $\mathrm{N}$ & 0 & $0.2(0.3)$ & & & & & \\
\hline Plantago myosuros Lam. & A & $\mathrm{N}$ & 2 & & & & & & \\
\hline $\begin{array}{l}\text { Malvella leprosa (Ortega) } \\
\text { Krapov. }\end{array}$ & $\mathrm{P}$ & $\mathrm{N}$ & 0 & & & & $1.7(2.9)$ & 33 & 1.00 \\
\hline
\end{tabular}


a)

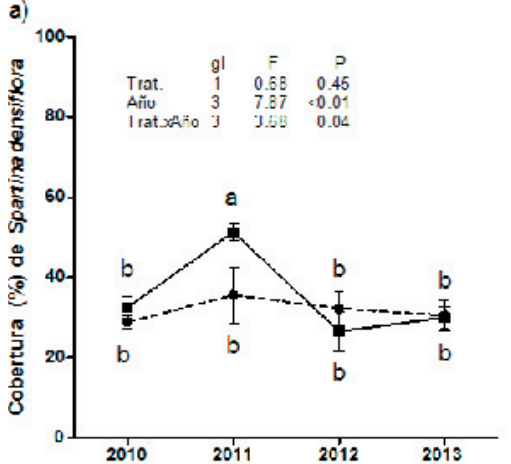

c)



e)

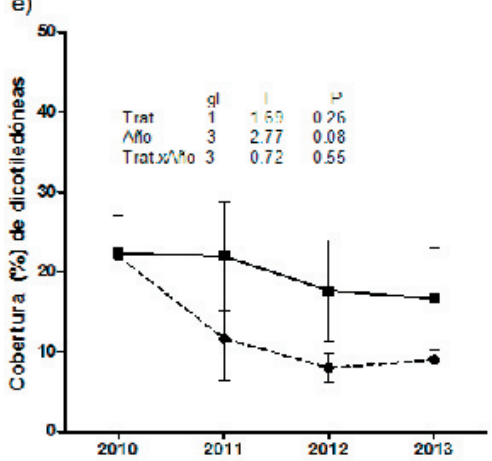

b)

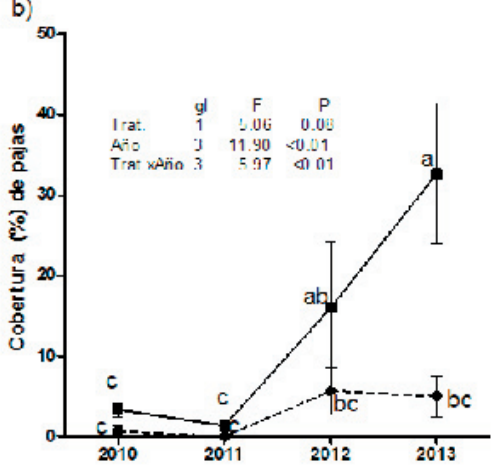

d)

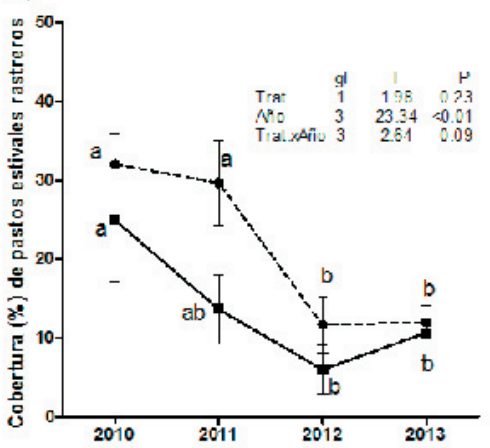

f)

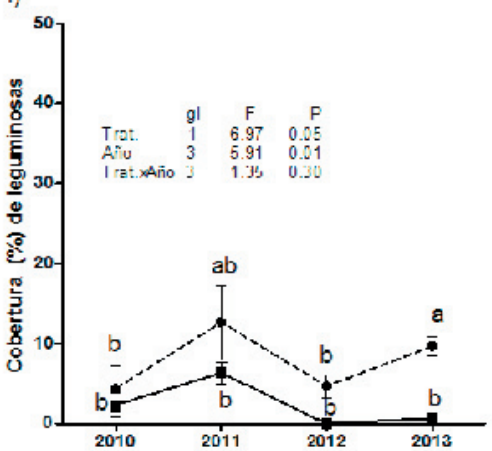

Figura 4. Evolución de la cobertura basal relativa (\%) de la especie dominante y de los grupos funcionales en los stands de espartillares bajo el tratamiento de defoliaciones cada 60 días entre octubre y mayo (línea punteada) y en el testigo no defoliado (línea entera) durante cuatro años consecutivos desde el inicio del tratamiento. Las barras verticales indican un error estándar $(1 \mathrm{EE})$ de la media. Se insertaron los resultados del análisis de varianza con un arreglo de medidas repetidas: F, grados de libertad (gl) y P para el factor principal tratamiento (Trat.), efecto intra-sujeto (medida repetida Año) e interacción entre el factor principal y el efecto intra-sujeto (Trat. $x$ Año). Letras distintas indican diferencias significativas surgidas de la prueba de Tukey.

Figure 4. Relative basal cover (\%) of functional groups in Spartina densiflora stands in the defoliation each 60-days from October to March treatment (dotted line) and in the not-defoliated control treatment (full line). Vertical lines indicate one standard error (1SE) of the mean. Insert: values of the repeated-measures analysis of variance: F, degree of freedom (gl) and P for the main factor (Trat.), within-subject factor (repeated measure Año) and interaction between the main and the within-subject factors (Trat. x Año). Different letters mean significant differences obtained by Tukey test.

experimental (Figura 4e), en tanto que la cobertura basal de las leguminosas fue superior bajo defoliaciones periódicas respecto del tratamiento testigo no defoliado a partir del año 2011 (Figura 4f).

Composición florística específica. La composición florística especifica relevada en los años 2010 y 2011 fue similar entre tratamientos $(T=0.99 ; P=0.84$ y $T=-0.57$; $P=0.26$, respectivamente). Las diferencias en la composición florística se expresaron en los relevamientos de los años 2012 y 2013 ( $T=-2.31$, $P=0.03$ y $T=-2.75 ; P=0.02$, respectivamente). Las especies responsables de las diferencias entre los stands defoliados y no defoliados entre 


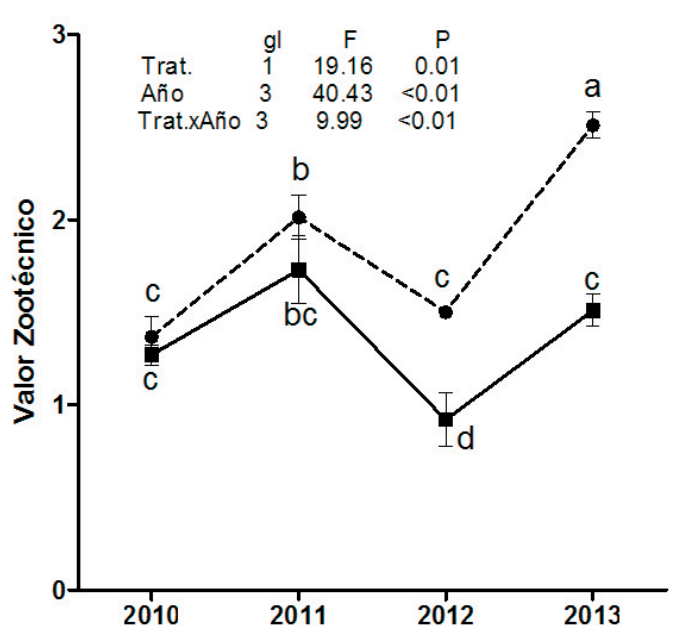

Figura 5. Valor forrajero de la vegetación de la comunidad del espartillar en los stands bajo el tratamiento de defoliaciones cada 60 días entre octubre y mayo (línea punteada) y el testigo no defoliado (línea entera). Las barras verticales indican un error estándar (1 EE) de la media. Se insertaron los resultados del análisis de varianza con un arreglo de medidas repetidas: F, grados de libertad (gl) y P para el factor principal tratamiento (Trat.), efecto intra-sujeto (medida repetida Año) e interacción entre el factor principal y el efecto intra-sujeto (Trat. x Año). Letras distintas indican diferencias significativas surgidas de la prueba de Tukey.

Figure 5. Forage quality index in the Spatina densiflora stands in the late summer defoliation followed by cattle exclusion from March to August treatment (dotted line) and under continuous cattle grazing treatment (full line). Vertical lines indicate one standard error (1 SE) of the mean. Insert: values of the repeated-measures analysis of variance: $\mathrm{F}$, degree of freedom (gl) and $\mathrm{P}$ for the main factor (Trat.), within-subject factor (repeated measure Año) and interaction between the main and the withinsubject factors (Trat. x Año). Different letters mean significant differences obtained by Tukey test.

octubre y mayo, identificadas a través de análisis de especies indicadoras y la prueba de Monte Carlo, fueron los pastos invernales nativos Elymus scabrifolius, Chaetotropis elongata y Phalaris angusta, y la leguminosa Melilotus officinalis, que aumentaron su contribución en los stands defoliados, en tanto que la paja nativa Cortaderia selloana disminuyó su cobertura en los stands defoliados (Tabla 2).

Valor forrajero. Las defoliaciones periódicas entre octubre y mayo incrementaron el valor zootécnico de la comunidad a partir del tercer ciclo sucesivo de cortes periódicos durante la estación de crecimiento (Figura 5).

\section{DisCUSIÓN}

El régimen de disturbios diseñado para cada una de las comunidades modificó la composición florística y mejoró la calidad de la oferta forrajera para el venado de las pampas, lo cual resultaría positivo para conservar esta especie. Estos resultados son consistentes con los modelos ecológicos que relacionan los disturbios con la conservación de la biodiversidad, y en particular con la hipótesis del disturbio intermedio, que propone que la máxima diversidad o mejor estado de conservación se alcanza a niveles intermedios de disturbios (Connell 1978; Pickett and White 1985; Milchunas et al. 1988; Westoby et al. 1989; Cingolani et al. 2005, 2008).

La defoliación intensa a fin del verano seguida por la exclusión al pastoreo del ganado entre marzo y agosto en los montes de tala del establecimiento lindero al Parque Nacional no sólo promovió la aparición o aumentó la contribución de los pastos nativos invernales Bromus catharticus, Bromusauleticusy Elymus scabrifolius, sino que también incrementó la cobertura de los pastos estivales erectos Bothriochloa laguroides y Paspalum dilatatum y de la leguminosa Melilotus officinalis, todas especies de alto valor nutritivo. La reinstalación de los pastos nativos invernales Bromus auleticus y Elymus scabrifolius en el campo ganado vecino luego de tres ciclos de exclusión al pastoreo del ganado entre marzo y agosto sugiere que la extinción local de especies nativas puede revertirse en tanto haya disponibilidad de propágulos (Bakker and Berendse 1999; Bossuyt and Honnay 2008), existan mecanismos de dispersión que permitan su arribo (Fenner and Thompson 2005) y disponibilidad de recursos e interacciones bióticas (i.e., competencia, facilitación) que aseguren su instalación. La existencia de individuos de Bromus auleticus y Elymus scabrifolius en el Parque (Fernández et al. 2004) habría asegurado la provisión de propágulos que pudieron arribar a las montes de tala de los lotes vecinos mediante diversos mecanismos dedispersión, tales comoel viento, los animales silvestres, el ganado, el tránsito de vehículos, entre otros. La defoliación intensa a fin de verano, al eliminar la biomasa aérea acumulada durante este período, habría relajado la competencia de las especies estivales sobre los propágulos de especies invernales, favoreciendo su germinación o brotación e instalación (Jacobo et al. 2000). Asimismo, la exclusión del pastoreo desde el principio de otoño evitó el consumo recurrente de estos grupos funcionales, que son los más preferidos por el ganado; esto aseguró su 
crecimiento y desarrollo (Jacobo et al. 2006). Luego de tres ciclos de exclusión al pastoreo vacuno entre marzo y agosto, las especies erectas que aumentaron su cobertura (pastos $\mathrm{C}_{3} \mathrm{y} \mathrm{C}_{4}$ ) llegarían cada primavera con una alta cantidad de biomasa acumulada, ejerciendo una fuerte competencia sobre las especies que evaden el pastoreo por su morfología (especies rastreras o de porte bajo) o por ser de bajo o nula preferencia por el ganado como las gramíneas Cynodon dactylon y Distichlis spicata y las dicotiledóneas Ambrosia tenuifolia, Centaurea melitensis, Malvella leprosa y Phyla canescens, que redujeron su cobertura relativa. Por lo tanto, la práctica aplicada modificó las interacciones bióticas y permitió la reinstalación de propágulos en un ambiente donde la disponibilidad de recursos ( $\mathrm{P}, \mathrm{MO}, \mathrm{CE}$, agua) no sería limitante para su establecimiento.

Se ha documentado que el venado de las pampas tiende a evitar aquellos lotes con presencia de ganado doméstico, pero suelen ocupar estos lotes una vez que se retira el ganado (Vila and Beade 1997; Dellafiore and Maceira 1998; Vila et al. 1998; Vila et al. 2008). En efecto, durante el período de exclusión al pastoreo vacuno, marzo a agosto, se registró la presencia de venados en los montes de tala, que los utilizaron como sitio de alimentación. Mientras tanto, no se observó presencia de venados en los montes de tala bajo pastoreo vacuno (Miñarro and Marino 2013). Este comportamiento refuerza lo sugerido por Vila et al. (2008) respecto de la facilitación como forma de interacción entre vacunos y venados que comparten recursos de alimentación. Se ha demostrado que los grandes herbívoros pueden facilitar el consumo a herbívoros pequeños mediante el consumo de volúmenes elevados de forraje, lo que promueve el rebrote de tejido de mayor calidad nutritiva y mejora la calidad de la oferta forrajera que queda disponible para los herbívoros de menor tamaño (McNaughton 1979). En este caso, las hembras adultas de razas británicas (que es la categoría que predomina en los rodeos de cría de esta región) alcanzan un peso vivo promedio de 420 a $480 \mathrm{~kg}$, consumen $\sim 10 \mathrm{~kg}$ MS/día y son poco selectivos, en tanto que el venado tiene un peso corporal promedio de $30 \mathrm{~kg}$, consume diariamente menos a un kilo de materia seca y es un herbívoro muy selectivo (Jackson and Giulietti 1988; Cosse Larghero 2002).

La defoliación periódica de la biomasa aérea del espartillar del Parque Nacional durante el períododecrecimientodelaespeciedominante, S. densiflora, modificó la composición florística de la comunidad: incrementó la contribución relativa de los pastos invernales nativos Elymus scabrifolius, Chaetotropis elongata y Phalaris angusta y de la leguminosa Melilotus officinalis, y disminuyó la cobertura de la paja nativa Cortaderia selloana. A pesar de que las defoliaciones por encima de los $25 \mathrm{~cm}$ eliminaron periódicamente tanto la biomasa de $S$. densiflora como la de la paja C. selloana, la cobertura relativa de $S$. densiflora no fue afectada, mientras que la de la paja disminuyó Esta especie presentaría una mayor capacidad de removilización de carbohidratos entre órganos aéreos y subterráneos y de producción de macollos (Di Bella et al. 2014b), lo que le permitiría mantener una cobertura constante en comparación con C. selloana. Asimismo, el régimen de defoliación implementado (cortes cada 60 días) habría producido un cambio en la estructura vertical del canope, relajando la competencia lumínica y favoreciendo a las especies de porte más bajo. La extensión del período de defoliaciones, entre octubre y mayo, aseguraría un ambiente con mayor disponibilidad de luz en otoño, que al coincidir con la disminución de la tasa de crecimiento de $S$. densiflora, relajaría la competencia por los recursos y permitiría la instalación y el establecimiento de los pastos y leguminosa invernales. Resultados similares se han documentado cuando mediante el pastoreo se remueve la biomasa área de especies estivales a fin de verano para favorecer la instalación y establecimiento de pastos invernales (Jacobo et al. 2000).

Tanto en el monte de tala como en el espartillar, las prácticas de manejo implementadas promovieron la aparecieron o aumentaron su contribución de pastos y leguminosas de alto valor nutritivo, en tanto que las especies que desaparecieron o disminuyeron su cobertura fueron herbáceas de porte bajo y escaso o nulo valor nutritivo. En consecuencia, este reemplazo de especies determinó un aumento del valor zootécnico de ambas comunidades, como se demostró en ambientes similares (Jacobo et al. 2006; Di Bella et al. 2014a), que beneficiaría tanto a los herbívoros silvestres como a los domésticos.

Las prácticas de manejo diseñadas para mejorar la oferta forrajera para la alimentación del venado de las pampas resultaron exitosas. Además, la aparición y el incremento de la cobertura de Elymus scabrifolius tanto en los montes de tala como en el espartillar, 
y el de Bromus auleticus en el monte de tala, son procesos muy alentadores para los objetivos de conservación de la flora nativa del Parque Nacional Campos del Tuyú ya que Elymus scabrifolius ha sido considerada en inminente peligro de extinción (Andrés 1986, citado en Abala et al. 2009), mientras que Bromus auleticus se encuentra en una forma fragmentada en la región central (Gutiérrez and Pensionar 2012), con algunas poblaciones sometidas a un proceso de reducción drástica de tamaño debido a la acción antrópica (Gutiérrez et al. 2013).

Agradecimientos. El trabajo fue financiado por la Secretaría de Ciencia y Técnica de la Universidad de Buenos Aires. Agradecemos a los Licenciados en Ciencias Ambientales María Lujan Linera y Santiago Mártir, quienes participaron de la toma de datos que formaron parte de sus tesis de grado, y a la estudiante María Pilar Repetto.

\section{REFERENCIAS}

Andrés, A. N. 1986. Variabilidad genética en poblaciones naturales de Agropyron scabrifolium (Doell) Parodi. Tesis de Maestría. Universidad Nacional de Rosario, Argentina.

APN. 2016. Administración de Parques Nacionales. Sistema de Información de Biodiversidad. www.sib.gov.ar. Revisado en febrero 2016

Augustine, D. J., D. T. Booth, S. E. Cox, and J. D. Derner 2012. Grazing intensity and spatial heterogeneity in bare soil in a grazing-resistant grassland. Rangeland Ecol Manage 65:39-46.

Bakker, J., and F. Berendse. 1999. Constraints in the restoration of ecological diversity in grassland and heathland communities. Trends Ecol Evol 13:63-68.

Bilenca, D., and F. Miñarro. 2004. Identificación de áreas valiosas de pastizal (AVPs) en las pampas y campos de Argentina, Uruguay y sur de Brasil. Fundación Vida Silvestre Argentina, Buenos Aires, Argentina.

Biondini, M., B. D. Patton, and P. E. Nyren.1988a. Grazing Intensity and Ecosystem Processes in a Northern MixedGrass Prairie, USA. Ecol Appli 8:469-479.

Biondini, M. E., P. W. Mielke Jr, and K. J. Berry. 1988b. Data-dependent permutation techniques for the analysis of ecological data. Vegetatio 75:161-168.

Bossuyt, B., and O. Honnay. 2008. Can the seed bank be used for ecological restoration? An overview of seed bank characteristics in European communities. J Veget Sci 19:875-884.

Burkart, S., R. J. C. León, and C. Movia. 1989. Inventario fitosociológico del pastizal de la Depresión del Salado (Prov. de Bs. As.) en un área representativa de sus principales ambientes. Darwiniana 30:27-69.

Bylo, L. N., N. Koper, and K. A. Molloy. 2014. Grazing intensity influences ground squirrel and american badger habitat use in mixed-grass prairies. Rangeland Ecol Manage 67:247-254.

Cabrera, A., and E. Zardini. 1978. Manual de la flora de los alrededores de Buenos Aires. 2a ed. ACME. Buenos Aires, Argentina. Pp. 755.

Cagnoni, M., and A. M. Faggi. 1993. La vegetación de la reserva de vida silvestre Campos del Tuyú. Parodiana 8: 101-112.

Cahuepé, M. A., M. Hidalgo, and A. Galatoire. 1985. Aplicación de índice de valoración zootécnica en pastizales de la Depresión del Salado. Rev Arg Prod An 5:681-690.

Cingolani, A. M., I. Noy-Meir, D. Renison, and M. Cabido. 2008. La ganadería extensiva, ¿es compatible con la conservación de la biodiversidad y de los suelos? Ecol Austral 18:253-271.

Cingolani, A. M., I. Noy-Meir, and S. Díaz. 2005. Grazing effects on rangeland diversity: a synthesis of contemporary models. Ecol Appl 15:757-773.

Connell, J. H. 1978. Diversity in Tropical Rain Forests and Coral Reefs. Science 199:1302-1310.

Cosse Larghero, M. 2002. Dieta y solapamiento de la población de venado (Ozotoceros bezoarticus) del campo "Los Ajos". Tesis de maestría, Universidad de la República, Uruguay.

Daget, P., and P. Poissonnet. 1971. Une méthode d'analyse phytosociologique des prairies. Critères d'application. Ann Agron 22:5-41.

Dellafiore, C. M., and N. Maceira. 1998. Problemas de conservación de los ciervos autóctonos de la Argentina. Mastozool Neotrop 5:137-145.

Deregibus, V. A., and A. Soriano. 1981. Los pastizales de la zona de cría de la Depresión del Salado desde el punto de vista ecológico. Rev Arg Prod Anim 1:60-83.

Deregibus, V. A., and M. A. Cahuepé. 1983. Pastizales naturales de la Depresión del Salado: Utilización basada en conceptos ecológicos. Revista de Investigaciones Agropecuarias 18:47-78.

Deregibus, V. A., E. J. Jacobo, and A. M. Rodríguez. 1995. Improvement in rangeland condition of the Flooding Pampa of Argentina through controlled grazing. Afr J Range Forage Sci 12:92-96.

Di Bella, C., E. J. Jacobo, R. A. Golluscio, and A. M. Rodríguez. 2014a. Effect of cattle grazing on soil salinity and vegetation composition along an elevation gradient in a temperate coastal salt marsh of Samborombón Bay (Argentina). Wetl Ecol Manag 22:1-13.

Di Bella, C., G. Striker, F. Escaray, F. Lattanzi, A. M. Rodríguez, and A. Grimoldi. 2014b. Saline tidal ooding effects on 
Spartina densiora plants from different positions of the salt marsh. Diversities and similarities on growth, anatomical and physiological responses. Environ Exp Bot 102:27-36.

Dufrene, M., and P. Legendre. 1997. Species assemblages and indicator species: the need for a flexible asymmetrical approach. Ecol Monogr 67:345-366.

Fenner, M., and K. Thompson. 2005. The ecology of seeds. Cambridge Press University, Cambridge, UK. Pp. 260.

Fernández, G. J., M. S. Beade, E. M. Pujol, and M. E. Mermoz. 2004. Plan de manejo de la Reserva de Vida Silvestre "Campos del Tuyu", General Lavalle, Provincia de Buenos Aires. Programa Pastizales Fundación Vida Silvestre Argentina con el apoyo de The Rufford Maurice Laing Foundation. Pp. 144.

Gutiérrez, G., and J. F. Pensiero. 2012. Bromus. Pp. 33-56 en F. O. Zuloaga, Z. E. Rugolo and A. M. Anton (eds.). Flora Argentina. Flora vascular de la Republica Argentina. Poaceae Gráficamente Ediciones, Córdoba, Argentina.

Gutiérrez, H. F., J. F. Pensiero, and J. M. Zabala. 2013. Effect of population combinations on the reproductive success and germination of seeds of Bromus auleticus (Poaceae). Grass Forage Sc 70:176-184.

Heitschmidt, R. K., and C. A. Taylor Jr. 1991. Livestock production. En: R. K. Heitschmidt and J. W. Stuth (eds.). Grazing management: an ecological perspective. Oregon: Timber Press. Pp. 259.

Isacch, J. P., M. S. Bó, V. M. Comparatore, L. P. Herrera, R. J. Vargas, and M. M. Martínez. 2001. Las aves de los pastizales costeros del sudeste de la Provincia de Buenos Aires. Pp. 269-285 en: Reserva de Biosfera Mar Chiquita: Características físicas, biológicas y ecológicas. Editorial Martín, Buenos Aires, Argentina.

Jackson, J. E., and J. D. Giulietti. 1988. The food habits of pampas deer Ozotoceros bezoarticus celer in relation to its conservation in a relict natural grassland in Argentina. Biol Conserv 45:1-10.

Jacobo, E. J., A. M. Rodríguez, J. L. Rossi, L. Salgado, and V. A. Deregibus. 2000. Rotational stocking and production of Italian ryegrass on Argentinean rangelands. J Range Manage 53:483-488.

Jacobo, E. J., A. M. Rodríguez, N. Bartoloni, and V. A. Deregibus. 2006. Rotational grazing effects on rangeland vegetation at a farm scale. Rangeland Ecol Manage 59:249-257.

Lavorel, S., S. McIntyre, J. Landsberg, and T. Forbes. 1997. Plant functional classifications: from general groups to specific groups based on response to disturbance. Trends Ecol Evol 12:474-478.

León, R., and M. Bertiller. 1982. Aspectos fenológicos de dos comunidades del pastizal de la Depresión del Salado (Prov. Bs. As.). Boletín de la Sociedad Argentina de Botánica 20:329-347.

León, R., S. Burkart, and C. Movia. 1979. Relevamiento fitosociológico del pastizal del norte de la Depresión del Salado. Instituto Nacional de Tecnología Agropecuaria. Serie Fitogeográfica 17:11-93.

León, R., G. Rusch, and M. Oesterheld. 1984. Pastizales pampeanos - impacto agropecuario. Phytocoenologia 12: 201-218.

McNaughton, S. J. 1979. Grazing as an optimization process: grass-ungulate relationships in the Serengeti. Amer Nat 11:691-703.

McNaughton, S. J. 1983. Compensatory plant growth as a response to herbivory. Oikos 40:329-336.

McNaughton, S. J., R. W. Ruess, and S. W. Seagle. 1988. Large mammals and process dynamics in African ecosystems. BioScience 38:794-800.

Mentesana, L. 2013 Calidad forrajera y selección de hábitat de forrajeo del venado de las pampas, (Ozotoceros bezoarticus celer) en Bahía de Samborombón, Pcia. de Buenos Aires. Tesis de Licenciatura. Universidad de Buenos Aires, Argentina.

Merino, M. L. 2003. Dieta y uso de hábitat del venado de las pampas, Ozotoceros bezoarticus celer Cabrera 1943, (Mammalia - Cervidae) en la zona costera de la Bahía de Samborombón, Buenos Aires, Argentina. Implicancias para su conservación. Tesis doctoral, Universidad Nacional de La Plata, Argentina.

Middleton, B. A. 1999. Wetland restoration, flood pulsing and disturbance dynamics. JohnWiley \& Sons, NewYork. Pp. 400.

Middleton, B. A. 2013. Rediscovering traditional vegetation management in preserves: Trading experiences between cultures and continents. Biol Conserv 158:271-279.

Milchunas, D. G., O. E. Sala, and W. K. Lauenroth 1988. A generalized model of the effects of grazing by large herbivores on grassland community structure. Amer Nat 132:87-106.

Miñarro F., and G. D. Marino (eds.). 2013. Ganadería Sustentable de Pastizal. Producir y conservar es posible. Aves Argentinas y Fundación Vida Silvestre Argentina, Buenos Aires, Argentina. Pp. 104.

Mueller-Dombois, D., and H. Ellenberg. 1974. Causal analytical inquiries into the origin of plant communities. Pp. 335-370 in: D. Mueller-Dombois \& H. Ellenberg (eds.). Aims and Methods of Vegetation Ecology. John Wiley and Sons, New York, USA.

Nasca, P. B. 2001. Fuego prescripto: efecto sobre la estructura y dinámica del espartillar de Spartina densiflora y su uso como herramienta de manejo para la conservación del venado de las Pampas. Tesis de Licenciatura, Universidad de Buenos Aires, Argentina.

Ojeda, R. A., Chillo, V., and G. B. Díaz ISENRATH. 2012. Libro rojo de mamíferos amenazados de la Argentina. Sociedad Argentina para el Estudio de los Mamíferos, Mendoza, Argentina.

Parera, A. F. 2003. Efectos del fuego sobre la fauna silvestre. Pp. 119-131 en: C. Kunst, S. Bravo and J. L. Panigatti (eds.). Fuego en los ecosistemas argentinos. Ediciones INTA.

Patton, B. D., X. Dong, P. E. Nyren, and A. Nyren. 2007. Effects of grazing intensity, precipitation, and temperature on forage production. Rangeland Ecol Manage 60:656-665. 
Pickett, S. T. A., and P. S. White. 1985. The ecology of natural disturbance and patch dynamics. Elsevier. Pp. 472.

Rey Benayas, J. M., A. Martins, J. M. Nicolau, and J. J. Schulz. 2007. Abandonment of agricultural land: an overview of drivers and consequences. Perspect Agr Vet Sci Nutr Nat Res 2:57.

Rodríguez, A. M., and E. J. Jacobo. 2012. Manejo de pastizales naturales para una ganadería sustentable en la Pampa Deprimida. Buenas Prácticas para una Ganadería Sustentable de Pastizal. Fundación Vida Silvestre Argentina - Aves Argentinas, Buenos Aires, Argentina.

Rodríguez, A. M., G. Roitman, M. L. Lipera, P. Preliasco, M. S. Beade, and F. Miñarro. 2012. Quemas prescriptas y defoliaciones mecánicas mejoran la oferta forrajera de los espartillares de la Bahía de Samborombón. Actas de la XXV Reunión Argentina de Ecología.

Sala, O., and J. Paruelo. 1997. Ecosystem services in grasslands. Pp. 237-251 in: G. Daily (ed.). Natural Services: Societal Dependence on Natural Ecosystems. Part III. Services supplied by major biomes. Island Press, Washington, USA.

Sala, O., M. Oesterheld, R. J. C. León, and A. Soriano. 1986. Grazing effects upon plant community structure in sub humid grasslands of Argentina. Vegetatio 67:27-32.

Sarmiento, G. 1996. Ecología de pastizales y sabanas en América Latina. Pp. 15-24 en: Biodiversidad y Funcionamiento de Pastizales y Sabanas en América Latina. CYTED and CIELAT, Mérida.

Savory, A. 1991. Holistic resource management: A conceptual framework for ecologially sound economic modelling. Ecol Econ 3:181-191.

Savory, A., and S. D. Parsons. 1980. The Savory grazing method. Rangelands 2:234-237.

Vecchio, M. C. 2014. Modificaciones en la vegetación y el suelo inducidos por el manejo del pastoreo en la estepa de halófitas de la Pampa Deprimida. Tesis de Maestría, Universidad de Buenos Aires, Argentina.

Vervoorst, F. 1967. Las comunidades vegetales de la depresión del Salado (Provincia de Buenos Aires). La vegetación de la República Argentina, Serie Fitogeográfica № 7, Instituto Nacional de Tecnología Agropecuaria, Buenos Aires.

Vila, A. R., M. S. Beade, and H. Pastore. 1998. Patrones de actividad del venado de las Pampas en "Campos del Tuyúu". Boletín Técnico, 43: 31. Fundación Vida Silvestre, Buenos Aires, Argentina.

Vila, A. R., M. S. Beade, and B. Barrios Lamunière. 2008. Home range and habitat selection of pampas deer. J Zool 276:95-102.

Vila, A. R., and M. S. Beade. 1997. Situación de la población del venado de las Pampas en la Bahía de Samborombón. Fundación Vida Silvestre Argentina, Buenos Aires, Argentina. Boletín Técnico 37:30.

Volesky, J. D., W. H. Schacht, and D. M. Richardson. 2004. Stocking rate and grazing frequency effects on nebraska sandhills meadows. J Range Manage 57:553-560.

Westoby, M., B. H. Walker, and I. Noy-Meir. 1989. Opportunistic management for rangelands not at equilibrium. J Range Manage 42:266-274.

Zabala, J. M., P. A. Tomas, G. E. Schrauf, and J. A. Giavedoni. 2009. Seed dormancy in Elymus scabrifolius (Döll) J.H. Hunz. Seed Sci \& Technol 37:248-251. 